Western University

Scholarship@Western

$2-1-2014$

\title{
Medial septal cholinergic neurons modulate isoflurane anesthesia.
}

Siew Kian Tai

Jingyi Ma

L Stan Leung

Follow this and additional works at: https://ir.lib.uwo.ca/physpharmpub

Part of the Medical Physiology Commons, and the Pharmacy and Pharmaceutical Sciences Commons

\section{Citation of this paper:}

Tai, Siew Kian; Ma, Jingyi; and Leung, L Stan, "Medial septal cholinergic neurons modulate isoflurane anesthesia." (2014). Physiology and Pharmacology Publications. 249.

https://ir.lib.uwo.ca/physpharmpub/249 


\title{
Medial Septal Cholinergic Neurons Modulate Isoflurane Anesthesia
}

\author{
Siew Kian Tai, Ph.D., Jingyi Ma, M.D., Ph.D., L. Stan Leung, Ph.D.
}

\section{ABSTRACT}

Background: Cholinergic drugs are known to modulate the response of general anesthesia. However, the sensitivity of isoflurane or other volatile anesthetics after selective lesion of septal cholinergic neurons that project to the hippocampus is not known.

Methods: Male Long Evans rats had 192 immunoglobulin G-saporin infused into the medial septum (n = 10), in order to selectively lesion cholinergic neurons, whereas control, sham-lesioned rats were infused with saline $(\mathrm{n}=12)$. Two weeks after septal infusion, the hypnotic properties of isoflurane and ketamine were measured using a behavioral endpoint of loss of righting reflex (LORR). Septal lesion was assessed by counting choline acetyltransferase-immunoreactive cells and parvalbuminimmunoreactive cells.

Results: Rats with 192 immunoglobulin G-saporin lesion, as compared with control rats with sham lesion, showed a 85\% decrease in choline acetyltransferase-immunoreactive, but not parvalbumin-immunoreactive, neurons in the medial septal area. Lesioned as compared with control rats showed increased isoflurane sensitivity, characterized by a leftward shift of the graph plotting cumulative LORR percent with isoflurane dose. However, lesioned and control rats were not different in their LORR sensitivity to ketamine. When administered with $1.375 \%$ isoflurane, LORR induction time was shorter, whereas emergence time was longer, in lesioned as compared with control rats. Hippocampal 62-100 Hz gamma power in the electroencephalogram decreased with isoflurane dose, with a decrease that was greater in lesioned $(n=5)$ than control rats $(n=5)$.

Conclusions: These findings suggest a role of the septal cholinergic neurons in modulating the sensitivity to isoflurane anesthesia, which affects both induction and emergence. The sensitivity of hippocampal gamma power to isoflurane appears to indicate anesthesia (LORR) sensitivity. (ANESthesiology 2014; 120:392-402)

C HOLINERGIC neurons have long been associated with consciousness and general anesthesia. Acetylcholine is one of the main neurotransmitters responsible for arousal. ${ }^{1,2}$ Acetylcholine is released in the neocortex and hippocampus during arousal, with maximal acetylcholine level during active waking. ${ }^{2-4}$ Acetylcholine in the cerebral cortex is derived from the basal forebrain, with the nucleus basalis innervating the neocortex and the medial septum and vertical limb of the diagonal band innervating the hippocampus and entorhinal cortex. ${ }^{5,6}$ Cholinergic neurons in the basal forebrain manifested maximal firing rates during active waking and paradoxical sleep ${ }^{7}$ and mediated an atropine-sensitive theta rhythm in the hippocampus, and a low-voltage, fast electrical activity in the neocortex. ${ }^{8}$

General anesthesia is associated with a decrease in cholinergic release and function. Different anesthetics induced a decrease in acetylcholine release in the cortex, ${ }^{9}$ whereas enhancement of brain acetylcholine level by anticholinesterase was shown to reduce the depth of anesthesia induced by propofol or isoflurane. ${ }^{10,11}$

Selective cholinergic neurotoxin 192 IgG-saporin lesion of the nucleus basalis prolonged the emergence from halothane, isoflurane, propofol, or pentobarbital anesethesia. ${ }^{12}$

\section{What We Already Know about This Topic}

- Acetylcholine is an important neurotransmitter in arousal, and increase in brain acetylcholine reduced anesthesia depth

- The contribution of the medial septal cholinergic neurons to isoflurane-induced unresponsiveness is unknown

What This Article Tells Us That Is New

- Selective lesion of cholinergic medial septal neurons enhanced sensitivity to isoflurane and prolonged isoflurane anesthesia

Laalou et al. ${ }^{13}$ reported that selective lesion of medial septal cholinergic neurons increased the anesthesia potency of propofol (>100 mg/kg intraperitoneal). It is not known whether selective cholinergic lesion of the medial septum alters the anesthesia response to volatile anesthetics.

We previously reported that inactivation or lesion of the medial septum prolonged the emergence time of both volatile and injectable general anesthetics. ${ }^{14,15}$ Electrolytic lesion of the medial septum also increased the sensitivity to intraperitoneal propofol and isoflurane. ${ }^{15}$ The medial septal area contains $\gamma$-aminobutyric acidergic and glutamatergic neurons, in addition to cholinergic neurons. ${ }^{16-18}$ To further investigate the specific involvement of the cholinergic neurons in modulating

Submitted for publication March 14, 2013. Accepted for publication July 26, 2013. From the Department of Physiology and Pharmacology (S.K.T., J.M., L.S.L.) and Program in Neuroscience (L.S.L.), The University of Western Ontario, London, Ontario, Canada.

Copyright $\odot$ 2013, the American Society of Anesthesiologists, Inc. Lippincott Williams \& Wilkins. Anesthesiology 2014; 120:392-402 
the hypnotic effects of isoflurane, we performed a selective lesion of the medial septal cholinergic neurons in Long Evans rats and studied the dose effects of isoflurane. We used the loss of righting reflex (LORR) as our primary measure for hypnosis because for a variety of anesthetics, the concentration that induces LORR in rats correlates highly with that of loss of consciousness in humans. ${ }^{19}$ We hypothesize that the anesthetic sensitivity to isoflurane is increased by septal cholinergic lesion, such that induction and emergence times are altered.

\section{Materials and Methods}

\section{Animals}

All procedures were approved by the Animal Care Committee of the University of Western Ontario (London, Ontario, Canada) and conducted according to the guidelines of the Canadian Council for Animal Care. Data were obtained from 26 adult male Long Evans rats (235-390g; Charles River Canada, St. Constance, Quebec, Canada). All animals were given water and regular rat chow ad libitum and housed under climate-controlled conditions with a 12-h light/dark cycle, and lights on at 7:00 Am. The temperature in the room was maintained at $21^{\circ}-23^{\circ} \mathrm{C}$.

\section{Lesion of Cholinergic Cells in the Medial Septum}

Cholinergic neurons in the medial septum were lesioned using a toxin 192 IgG-saporin (Advanced Targeting Systems, San Diego, CA) specific for cholinergic neurons that express p75 receptors. ${ }^{20,21} \mathrm{~A}$ rat was anesthetized with sodium pentobarbital $(60 \mathrm{mg} / \mathrm{kg}$ intraperitoneal). $192 \mathrm{IgG}$-saporin was diluted with sterile saline, loaded into a Hamilton syringe, and infused bilaterally into the medial septum (anterior to bregma $0.5 \mathrm{~mm}$, lateral to midline $(\mathrm{L}) \pm 0.5 \mathrm{~mm}^{22}$ ). At each track, the 30 -gauge cannula was first lowered to $5.7 \mathrm{~mm}$ ventral from the dura $(\mathrm{V})$, and then to $\mathrm{V} 7.8 \mathrm{~mm} .192 \mathrm{IgG}$-saporin $(0.35 \mu \mathrm{g} / \mu \mathrm{l} ; 0.3 \mu \mathrm{l}$ at $\mathrm{V} 5.7 \mathrm{~mm}, 0.4 \mu \mathrm{l}$ at $\mathrm{V} 7.8 \mathrm{~mm})$ was infused at a constant rate of $0.5 \mu \mathrm{l} / 10 \mathrm{~min}$ using an infusion pump (Harvard Apparatus, South Natick, MA). After each infusion, the needle remained in place for $10 \mathrm{~min}$. Shamlesioned rats were infused with equal volumes of saline.

\section{Electrode Implantation}

192 IgG-saporin and saline-infused rats were implanted with depth electrodes immediately or 2 days after medial septum infusion. Under sodium pentobarbital $(60 \mathrm{mg} / \mathrm{kg}$ intraperitoneal) anesthesia, electrodes were placed in the CA1 stratum radiatum of the dorsal hippocampus (posterior to bregma $3.8 \mathrm{~mm}$, lateral to midline $2.8 \mathrm{~mm}$; ventral to dura $3.1 \mathrm{~mm}$ ) and layer IV of the frontal cortex (anterior to bregma $2 \mathrm{~mm}$, lateral to midline $3 \mathrm{~mm}$, ventral to dura $1.5 \mathrm{~mm}$ ); coordinates were from the Rat Brain Atlas of Paxinos and Watson. ${ }^{22}$ Each electrode comprised a $125-\mu \mathrm{m}$ stainless steel wire insulated with Teflon, except at the cut tip. A jeweller's screw was placed epidurally over the cerebellum. All electrodes and screws were fixed on to the skull with dental cement. Each electroencephalogram signal was recorded using one active electrode (frontal cortex or hippocampus) with respect to the cerebellar screw that served as both reference and ground.

\section{Anesthetic and Electroencephalogram Response to Isoflurane in 192 lg G-saporin/sham-lesioned Rats}

Loss of righting reflex was used as the behavioral endpoint to investigate the hypnotic properties of isoflurane (Forane; Baxter Corporation, Mississauga, Ontario, Canada), following previously described methods with slight modifications. ${ }^{23}$ To determine the isoflurane concentration that induced LORR, each rat was placed in a small acrylic glass chamber $(23 \times 12 \times 12 \mathrm{~cm} 3)$ connected to an isoflurane vaporizer with $1 \mathrm{l} / \mathrm{min}$ flow of $100 \%$ oxygen. The outflow from the chamber was connected to an infrared gas analyzer (RGM5250; Ohmeda, Louisville, CO). Isoflurane was administered to the chamber starting at $0.5 \%$ concentration, and was incremented by $0.125 \%$ until LORR occurred in the rat. The concentration of isoflurane was shown to reach a plateau in less than $10 \mathrm{~min}$ after a change of dose, as verified by the infrared gas analyzer. Each concentration of anesthetic was maintained for a minimum equilibration period of $15 \mathrm{~min}$, after which the chamber was rotated to place the rat on its back. A rat was considered to show LORR if it did not turn onto all four feet within $30 \mathrm{~s}$, and this was confirmed by a subsequent trial. The percentage of rats showing LORR at each dose of isoflurane was established for $192 \mathrm{IgG}$-saporin lesioned and sham-lesioned rats, and the ED50 was estimated from the dose-response equation described in Statistical Analysis.

In five 192 IgG-saporin lesioned rats and five shamlesioned rats, electroencephalogram recordings were obtained from the hippocampus and frontal cortex at different isoflurane concentrations while the rat was inside the small acrylic glass chamber described above. The frontal and hippocampal electrodes were connected to two different channels of a telemetry device that transmitted radio waves to a receiver approximately $0.5 \mathrm{~m}$ above the chamber (W5 Telemetry System; Triangle BioSystems, Durham, $\mathrm{NC}$ ). The received signals were then digitized ${ }^{24}$ as described in Statistical Analysis. Before the delivery of isoflurane, baseline electroencephalogram recordings were made in a larger chamber during awake immobility (head was held up and frontal electroencephalogram showed low-voltage fast activity) and during walking (when rat walked, turned its body, and reared). Electroencephalogram recording was made for $2 \mathrm{~min}$, starting $13 \mathrm{~min}$ after each isoflurane concentration or increment of $0.125 \%$, ending with $0.125 \%$ above the isoflurane concentration that induced LORR.

In order to assess isoflurane induction and emergence, the time to LORR and time for recovery of righting were investigated after $1.375 \%$ isoflurane was given. Each rat was individually placed in the small acrylic glass chamber, after which $1.375 \%$ isoflurane was delivered with $1 \mathrm{l} / \mathrm{min}$ oxygen. At 15-s intervals, the chamber was rotated to place a rat on its back, and the ability of a rat to right itself was assessed. The time to LORR (induction time) was defined as the time 
at which a rat first demonstrated a loss of righting for more than $30 \mathrm{~s}$, from the time of isoflurane onset. After 30-min exposure to $1.375 \%$ isoflurane, the rat was removed from the chamber and placed in a supine position in room air. The emergence time was defined as the time a rat righted itself, landing all four feet on the floor, from the time of removal from the isoflurane chamber.

\section{Anesthetic Response to Ketamine in 192 IgG-saporin/ sham-lesioned Rats}

Anesthetic responses to ketamine were also studied using cumulative intraperitoneal doses, as described elsewhere. ${ }^{25}$ The experimenter was not aware of the treatment history of the rat. A rat was given an initial dose of $40 \mathrm{mg} / \mathrm{kg}$ of ketamine intraperitoneally, and then nine increments of $20 \mathrm{mg} /$ $\mathrm{kg}$ were injected intraperitoneally at 10 -min intervals until it reached a final dose of $220 \mathrm{mg} / \mathrm{kg}$ intraperitoneally. Righting was tested by placing a rat in a supine position, and LORR was defined if the rat was unable to turn from the supine position to land on its feet, in two trials separated by $30 \mathrm{~s}$. Typically, a dose of ketamine that induced LORR, as defined, did not abolish neck and body movements in the supine position.

\section{Histology}

At the end of the experiments, rats were deeply anesthetized with pentobarbital and perfused through the heart with $400 \mathrm{ml}$ of cold saline followed by $500 \mathrm{ml}$ of cold $4 \%$ paraformaldehyde solution in $0.1 \mathrm{M}$ phosphate buffer (PB; $\mathrm{pH} 7.4$ ). The brain was removed and postfixed in the latter solution at $4^{\circ} \mathrm{C}$. Choline acetyltransferase and parvalbumin immunocytochemistry were carried out on coronal sections through the medial septum. ${ }^{21} \mathrm{~A}$ freezing microtome was used to section the medial septum at $40 \mu \mathrm{m}$ within $12 \mathrm{~h}$ of fixing.

For the choline acetyltransferase and parvalbumin staining, the brain sections were first incubated in $1 \%$ sodium borohydride in $0.1 \mathrm{M} \mathrm{PB}$ for $15 \mathrm{~min}$ and subsequently rinsed in PB. To block nonspecific labeling, the brain sections were incubated in 10\% normal goat serum (Sigma-Aldrich, St. Louis, MO) in $0.1 \mathrm{M} \mathrm{PB}$ containing $0.1 \%$ Triton X-100 (Sigma-Aldrich) for $1 \mathrm{~h}$ at room temperature. The sections were rinsed briefly in $\mathrm{PB}$ and incubated at $4^{\circ} \mathrm{C}$ for $48 \mathrm{~h}$ in primary antibody solution containing mouse monoclonal choline acetyltransferase (1:200; Cedarlane, Burlington, Ontario, Canada) or parvalbumin (1:100; Sigma-Aldrich) in $1 \%$ normal goat serum. Sections were rinsed in three changes of $\mathrm{PB}$ and followed by incubation in biotin-conjugated goat antimouse secondary antiserum (1:200; Jackson ImmunoResearch, West Grove, PA) for $1 \mathrm{~h}$ in room temperature. The sections were then rinsed several times in PB. Avidin Biotin Complex solution (Vector Laboratories, Burlington, Ontario, Canada) was prepared $20 \mathrm{~min}$ before use by adding equal volumes of solutions A and B in PB (1:1:100). The sections were incubated in the Avidin Biotin Complex solution for $1 \mathrm{~h}$ at room temperature. After three washes in $\mathrm{PB}$, the sections were incubated in a solution containing $0.05 \%$ diaminobenzidine tetrahydrochloride (Sigma-Aldrich) and 0.03\% hydrogen peroxide in $\mathrm{PB}$ at room temperature in a fume hood until they reached the desired color intensity $(1-3 \mathrm{~min})$. The sections were then rinsed several times in $\mathrm{PB}$ and mounted on glass slides. Finally, they were dehydrated in a series of 70 , 95, and $100 \%$ ethyl alcohol, cleared in xylene $(5 \times 2 \mathrm{~min})$ and coverslipped with DePex (BDH; VWR International, Mississauga, Ontario, Canada) mounting medium.

The number of choline acetyltransferase- and parvalbumin-immunoreactive cells was counted in three representative coronal sections at anterior $(\neg \mathrm{A} 0.7 \mathrm{~mm})$, middle $(-\mathrm{A}$ $0.4 \mathrm{~mm})$, and posterior $(\neg \mathrm{A} 0.2 \mathrm{~mm})$ levels of the medial septum-diagonal band of Broca region. Images of selected sections were captured with a digital camera attached to the microscope. Choline acetyltransferase-immunoreactive cells were counted from digital images with $\times 40$ magnification, with the medial septal area bounded mediolaterally by vertical lines through the tips of the lateral ventricles, and dorsally by the corpus callosum, as shown in figure 1, A and B. Parvalbumin-immunoreactive cells were counted from images of $\times 100$ magnification, in a $1.23 \times 1.64 \mathrm{~mm}^{2}$ rectangle (the counting frame was about double the size of fig. 1, $\mathrm{C}$ and $\mathrm{D}$ ). Each image was counted by at least two persons who were blinded to the treatment history of the images. The number of immunoreactive cells for each rat was the average of counts in the three sections. Recording electrode placements in the hippocampus and frontal cortex were histologically verified in $40-\mu \mathrm{m}$ thionin-stained brain sections.

\section{Animals and Sequence of Experiments}

Twelve rats were infused with $192 \mathrm{IgG}$-saporin, and 12 rats were infused with saline in the medial septum. For all rats, the first experiment started 14-17 days after infusion of 192 IgG-saporin or saline, in which LORR to different isoflurane doses was assessed; electroencephalogram was also recorded in five rats of each group. The second experiment was done 6-10 days later to record the onset and duration of LORR after $1.375 \%$ isoflurane. Ten to 14 days later, the behavioral responses to cumulative doses of ketamine were assessed in the last experiment (five rats in each group). Rats were perfused at 35-42 days after lesion. Of the 12 lesioned rats, only 10 rats with confirmed choline acetyltransferase immunocytochemistry were included in the results; one rat was not adequately perfused, and another died unexpectedly before perfusion. Of the 12 saline-infused (sham-lesioned) rats, immunocytochemistry was performed in 10 rats. Because it is not expected that control rats should be deficient in choline acetyltransferaseimmunoreactive neurons, all 12 sham-lesioned rats in the ED50 determination were analyzed, including two without choline acetyltransferase immunostaining. Choline acetyltransferase-immunoreactive counts were available for all control rats used in the experiments involving $1.375 \%$ isoflurane $(\mathrm{n}=6)$, electroencephalogram recording $(\mathrm{n}=5)$, and ketamine $(\mathrm{n}=5)$. In addition, two intact, unoperated rats were also used in the $1.375 \%$ isoflurane experiment. 


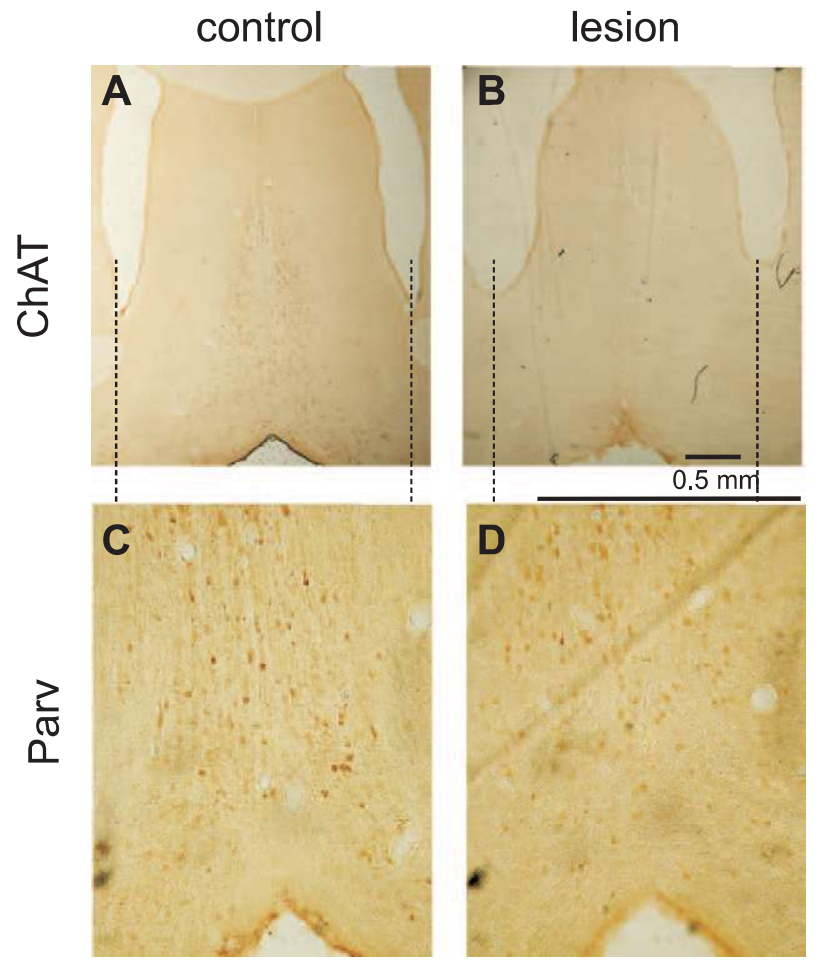

Fig. 1. Representative histological sections of the medial septal area stained for immunoreactivity to choline acetyltransferase (ChAT; $A$ and $B$ ) and parvalbumin (Parv; $C$ and $D$ ). Right column, control sham-lesioned rat; left column, 192 IgGsaporin lesioned rat. Dashed vertical lines in $A$ and $B$, drawn through the ventral tip of the lateral ventricle, indicate extent of medial septal cell counting. Horizontal line, $0.5 \mathrm{~mm}$ calibration; same for $A$ and $B$, and for $C$ and $D$.

\section{Statistical Analysis}

Power spectral analysis of the electroencephalogram was performed by custom software. ${ }^{24}$ Electroencephalogram was sampled at $1 \mathrm{kHz}$, and five consecutive samples were averaged and stored at every $5 \mathrm{~ms}$ to give an effective $200 \mathrm{~Hz}$ sampling frequency of the electroencephalogram signal that was passed through a low-pass digital filter with a $3 \mathrm{~dB}$ drop-off point at $90 \mathrm{~Hz}$. Artifact-free electroencephalographic signals were selected by eye. Each electroencephalogram segment consisted of 1,024 points $(5.12 \mathrm{~s}$ ), and one tenth of the total segment length was tapered by a cosine function $1 / 2(1-\cos$ $(10 \pi t / T))$ where $t$ is time from each end and $T$ is the segment length. After fast Fourier transform, the spectral values were smoothed by an elliptical window falling off at $\left[1-\left(f_{\mathrm{i}} \mid f_{\mathrm{m}}\right)^{2}\right]^{0.5}$, where $f_{\mathrm{i}}=$ deviation from the center frequency and $f_{\mathrm{m}}=$ half the smoothing bandwidth $=2$. Effectively, the frequency resolution is $0.195 \mathrm{~Hz}$ (reciprocal of $5.12 \mathrm{~s}$ ), and after smoothing $\left(2 \times f_{\mathrm{m}}+1=5\right.$ frequency bins $)$, statistically independent values were $0.98 \mathrm{~Hz}$ apart. Each power spectral value derived from one segment is estimated to have 10 degrees of freedom (df), and the average spectrum of each condition consisted of 6-12 segments, that is, 60-120 df. The average integrated power of the electroencephalogram for selected frequency bands at the frontal cortex and hippocampus electrodes was determined by averaging the power across frequency bins within the range of delta $(0.8-4 \mathrm{~Hz})$, beta $(12-30 \mathrm{~Hz})$, low gamma $(\gamma 1,30-58$ $\mathrm{Hz})$, and high gamma $(\gamma 2,62-100 \mathrm{~Hz})$. In addition, hippocampal theta power was integrated from 7 to $8 \mathrm{~Hz}$, and frontal "theta" power was integrated from 4 to $12 \mathrm{~Hz}$. Power was determined in logarithmic units, and $7 \log$ units of power were equivalent to a $1 \mathrm{mV}$ peak-to-peak sinusoidal wave. For evaluation of the dose-response to isoflurane of each rat, the difference in power from baseline immobility was calculated for each frequency band (delta, theta, beta, low and high gamma) at a particular isoflurane dose.

GraphPad Prism software version 4.0 (GraphPad Prism, Inc., San Diego, CA) and GB Stat (Dynamic Microsystems Inc., Silver Spring, MD) and SAS 9.1.3 (SAS Institute Inc., Cary, NC) were used for statistical evaluation. LORR doseresponse data were curve-fitted by nonlinear regression with Prism to give the half maximal effective concentration-dose values $(\mathrm{ED} 50 \pm \mathrm{SEM})$ with the equation: $\mathrm{Y}=\mathrm{Y}_{\min }+\left(\mathrm{Y}_{\max }-\right.$ $\left.\mathrm{Y}_{\min }\right) /[1+10 \log (\mathrm{ED} 50-\mathrm{X}) \times \mathrm{m}]$, where $\mathrm{Y}$ is the percentage of the population showing LORR; $Y_{\text {min }}$ and $Y_{\text {max }}$ are the minimal and maximal values of $Y$, respectively; ED50 is the drug dose for a half $\left(\mathrm{Y}_{\max }-\mathrm{Y}_{\min }\right), \mathrm{X}$ is the logarithmic drug dose, and $\mathrm{m}$ is the Hill slope constant. The $F$-test for nonlinear regressions was then used to determine whether the calculated ED50 was significantly different between groups. Group differences in cell count, onset of LORR, return of righting reflex, and electroencephalogram power were compared using unpaired Student $t$ test (GB Stat). The difference between electroencephalogram power during baseline immobility and walking was tested by determining the mean and SEM, and the statistical significance of the deviation from zero as tested by $t$ test (GB Stat). For the effect of isoflurane dose on electroencephalogram power in lesioned and control rats, a two-factor repeated-measures ANOVA with Greenhouse-Geisser adjustment (SAS 9.1.3) was used; one factor was group (between lesioned and control subjects) and the other was dose (within-subjects repeated measures). Data were expressed as mean \pm SEM, with $95 \%$ CIs. $P$ value less than 0.05 (two-tailed) was considered to be statistically significant.

\section{Results}

\section{IgG-saporin Depleted Cholinergic Neurons in the Medial Septal Area}

As assessed by the number of choline acetyltransferaseimmunoreactive cells (fig. 1, A and B), cholinergic neurons in the medial septum were greatly reduced by bilateral infusions of 192 IgG-saporin in the medial septum. The average number of choline acetyltransferase-immunoreactive neurons per coronal section in $192 \mathrm{IgG}$-saporin lesioned rats was $23.8 \pm 5.5(95 \% \mathrm{CI}, 11.3-36.3, \mathrm{n}=10)$, which was reduced by $85 \%$ as compared with that in control (sham-lesioned) rats $(158.9 \pm 22.4,95 \% \mathrm{CI}, 108.2-209.5, \mathrm{n}=10, P=0.002$, $t$ test). In contrast, the number of parvalbumin-immunoreactive neurons (fig. 1, C and D) was not significantly different between lesioned $(57.8 \pm 10.3,95 \%$ CI, 34.1-81.5, 
$\mathrm{n}=9)$ and control rats $(79.1 \pm 7.1,95 \% \mathrm{CI}, 62.7-95.5$, $\mathrm{n}=9 ; P>0.13, t$ test).

\section{Medial Septum Cholinergic Lesion Enhanced Anesthetic Sensitivity to Isoflurane}

We first examined whether the anesthetic sensitivity of isoflurane was altered in 192 IgG-saporin lesion rats by using a protocol of increasing isoflurane concentration starting at $0.375 \%$ and ending at $0.125 \%$ above the concentration after LORR occurred. No rats showed LORR at $0.5 \%$ or lower isoflurane concentration. However, at $0.625 \%$ isoflurane, 7 of the 10 lesioned rats, but none of the 12 control (sham-lesioned) rats, showed LORR. At $0.75 \%$, all lesioned rats but only half of the control rats showed LORR. The percent LORR versus isoflurane dose-response graph was leftward shifted for lesioned as compared with that of control rats (fig. 2A), indicating a higher isoflurane sensitivity in lesioned than control rats. The concentration at which half the rats showed LORR, or ED50 (LORR), was $0.62 \%$ in lesioned rats (95\% CI, $0.59-0.64 \%$, $\mathrm{n}=10)$ and $0.74 \%$ in control rats $(95 \% \mathrm{CI}, 0.71-0.76 \%$, $\mathrm{n}=12$ ). The ED50 (LORR) was significantly different between lesioned and control rats $(P<0.0001)$.

When exposed to $1.375 \%$ isoflurane, $192 \mathrm{IgG}$-saporin lesioned rats showed a significantly shorter LORR onset (induction) time averaging $5.22 \pm 0.2 \mathrm{~min}(95 \% \mathrm{CI}, 4.76-$ $5.67 \mathrm{~min}, \mathrm{n}=10)$ as compared with $8.22 \pm 0.41 \mathrm{~min}(95 \%$ CI, 7.16-9.29 min, $\mathrm{n}=6)$ in control (sham-lesioned) rats $(P<$ $0.005, t$ test; fig. $2 \mathrm{~B}$ ). When returned to room air after $30 \mathrm{~min}$ of exposure to $1.375 \%$ isoflurane, the time to recover righting reflex (emergence time) was significantly longer in lesioned rats $(8.65 \pm 0.76 \mathrm{~min}, 95 \% \mathrm{CI}, 6.92-10.37 \mathrm{~min}, \mathrm{n}=10)$ as compared with control rats $(4.15 \pm 0.8 \mathrm{~min}, 95 \% \mathrm{CI}, 2.1-6.19 \mathrm{~min}$, $\mathrm{n}=6 ; P<0.005, t$ test; fig. $2 \mathrm{~B})$. The sham-lesioned rats $(\mathrm{n}=6)$ were not distinguishable in their induction or emergence time from intact unoperated rats of a similar age $(\mathrm{n}=2)$.

\section{Hippocampal Gamma Electroencephalogram Power Decreased with Isoflurane Concentration}

Hippocampal and frontal electroencephalograms were wirelessly recorded during baseline awake-immobility and walking before isoflurane, and after incremental isoflurane doses until LORR ensued. Low-voltage fast activity was recorded in the frontal cortex and irregular slow activity in the hippocampus during baseline immobility (fig. 3A). After the first dose of isoflurane at $0.5 \%$, clear increase in slow activity in the frontal cortex and decrease in fast activity in the both the frontal cortex and hippocampus were observed (fig. 3A), with no apparent difference shown between the representative control and lesioned rats. Power spectral analysis confirmed an increase in slow delta power in the frontal cortex (fig. 3B) and a decrease in gamma activity $(30-100 \mathrm{~Hz})$ in both the frontal cortex and hippocampus (fig. 3, B and C).

We studied whether electroencephalogram power at particular frequency bands were different between immobility and walking in the freely moving rat. Because lesioned and
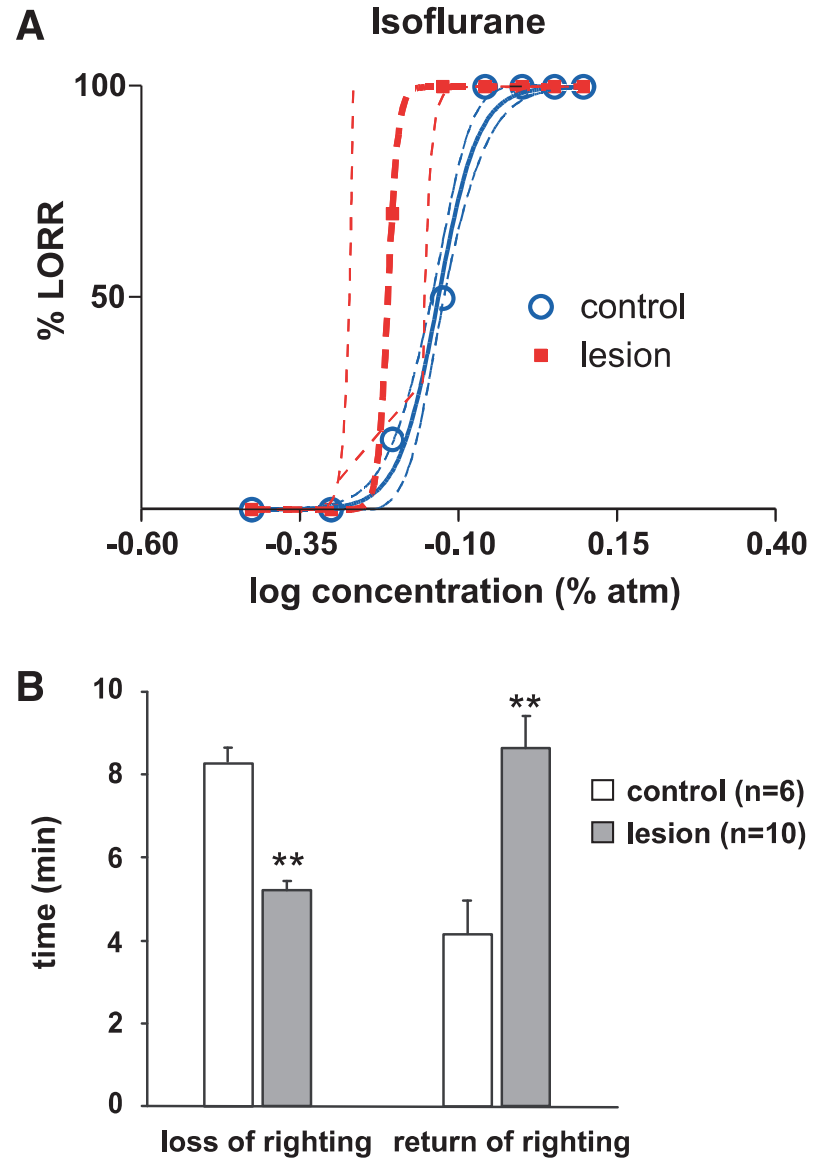

Fig. 2. Septal cholinergic lesioned rats and sham-lesioned (control) rats were different in isoflurane sensitivity, induction, and emergence time. $(A)$ Dose-response plot of cumulative percentage of rats showing loss of righting reflex (\% LORR) was different between lesioned $(n=10)$ and control $(n=12)$ rats. Horizontal axis is logarithmic dose of isoflurane in percentage atmosphere concentration ( $\%$ atm); dashed traces are $95 \%$ confidence limits of the thick regression trace. $(B)$ Induction and emergence time with exposure to $1.375 \%$ isoflurane. Time to LORR (induction time) was significantly shorter, and time to return to righting (emergence time) after $30 \mathrm{~min}$ in $1.375 \%$ isoflurane was significantly longer, in lesioned than in control rats. Lesioned rats $(n=10)$ compared with control rats $(\mathrm{n}=6),{ }^{\star \star} P<0.005$, unpaired $t$ test.

control groups of rats ( $\mathrm{n}=5$ in each group) did not differ significantly in the integrated power at any frequency band, data from all 10 rats were combined to test the statistical significance of the power difference between walking and baseline immobility (table 1). Hippocampal but not frontal delta power was significantly different between awake immobility and walking (fig. 4A; table 1). Although the rhythmic hippocampal theta rhythm was larger during walking (not shown) than immobility, $4,7,8,24$ the difference was not statistically significant for the integrated theta power (table 1; fig. 4A), which did not optimally isolate the oscillatory from the irregular slow activity. ${ }^{24}$ Also, immobility-related hippocampal theta rhythm was very small during baseline immobility or with isoflurane $(\geq 0.5 \%)$ anesthesia, in control or 


\section{Control 2. Lesion}

\section{A Immobility baseline}
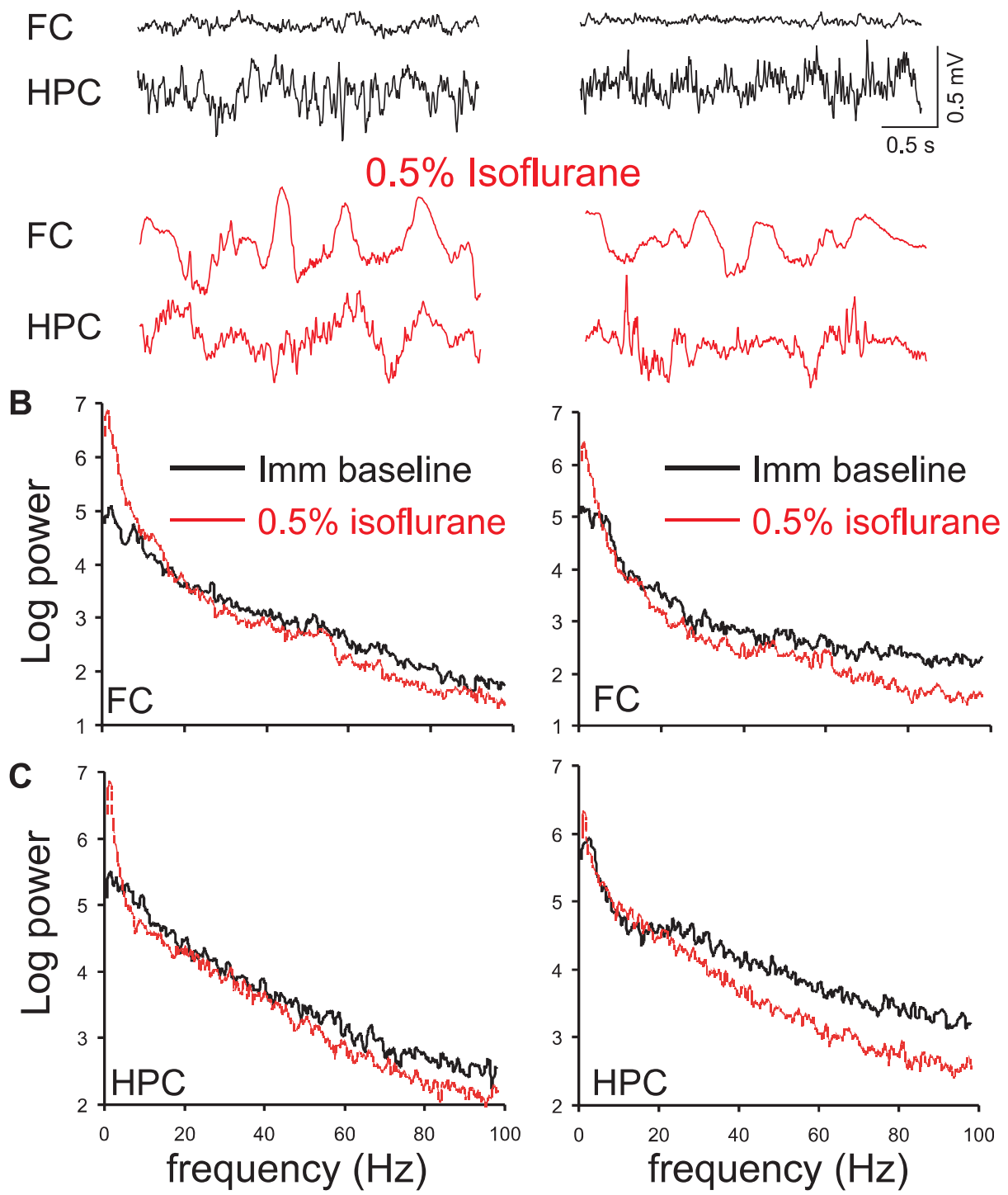

Fig. 3. Representative electroencephalogram and power spectra recorded in control sham-lesioned rats (column 1) and 192 immunoglobulin G-saporin lesioned rats (column 2). (A) Electroencephalogram traces derived from electrodes in the frontal cortex (FC) and hippocampus (HPC), during immobility baseline and $15 \mathrm{~min}$ after $0.5 \%$ isoflurane. (B) Power spectra of frontal cortical electroencephalogram after $0.5 \%$ isoflurane overlaid on spectra during immobility baseline. $(C)$ Power spectra of hippocampal electroencephalogram, same format as $B$. Note general decrease of high-frequency $(>50 \mathrm{~Hz})$ gamma power at FC and HPC at $0.5 \%$ isoflurane compared with immobility baseline, with larger decrease in lesioned than in control rat.

lesioned rats. Beta power was not different between immobility and walking, in the frontal or hippocampal electroencephalogram, in control or lesioned rats (fig. 4B). Higher gamma electroencephalogram power in the hippocampus and frontal cortex was found during walking as compared with awake immobility, generally for both low $(\gamma 1)$ and high gamma $(\gamma 2)$ power (fig. $4 \mathrm{C}$; table 1$)$.

Electroencephalogram was recorded and analyzed at 13$15 \mathrm{~min}$ after an increase in isoflurane dose, and the change in logarithmic electroencephalogram power from baseline awake immobility was calculated for the different frequency bands.
The frontal delta power increased after the initial dose of $0.5 \%$ isoflurane (fig. 3B), but was not further increased with higher doses of isoflurane (fig. 4A). There was also no statistical difference between the frontal delta power change in lesioned and control rats (table 1). Frontal theta (4-12 Hz) and beta $(13-30 \mathrm{~Hz})$ power were not significantly affected by isoflurane dose (fig. 4, A and B). Frontal $\gamma 1$ and $\gamma 2$ changes were not significantly different between lesioned and control groups, or significantly dependent on isoflurane dose (table 1). The mean frontal $\gamma 2$ power decreased, whereas mean $\gamma 1$ power increased with isoflurane dose from 0.5 to $0.75 \%$ (fig. $4 \mathrm{C}$ ). 
Table 1. Statistical Significance of Difference in Integrated Power of Different EEG Frequency Bands in the Frontal Cortex and Hippocampus

\begin{tabular}{|c|c|c|c|c|c|c|c|c|c|c|}
\hline \multirow{3}{*}{$\begin{array}{c}\text { Frequency } \\
\text { Band }\end{array}$} & \multicolumn{5}{|c|}{ Frontal Cortex } & \multicolumn{5}{|c|}{ Hippocampus } \\
\hline & \multirow{2}{*}{$\begin{array}{c}\text { (Walk-Imm) } \\
t(9)\end{array}$} & \multicolumn{2}{|c|}{ Group } & \multicolumn{2}{|c|}{ Dose } & \multirow{2}{*}{$\begin{array}{c}\text { (Walk-Imm) } \\
t(9)\end{array}$} & \multicolumn{2}{|c|}{ Group } & \multicolumn{2}{|c|}{ Dose } \\
\hline & & $F(1,8)$ & $P$ Value & $F(2,16)$ & $P$ Value $†$ & & $F(1,8)$ & $P$ Value & $F(2,16)$ & $P$ Value $†$ \\
\hline $\begin{array}{l}\text { Delta } \\
(0-4 \mathrm{~Hz})\end{array}$ & -1.54 & 0.10 & 0.76 & 0.26 & 0.66 & $-3.22 \S$ & 0.58 & 0.36 & 1.21 & 0.32 \\
\hline $\begin{array}{l}\text { Theta* } \\
(4-12 \mathrm{~Hz}) \\
(7-8 \mathrm{~Hz})\end{array}$ & -2.06 & 0.25 & 0.63 & 0.39 & 0.62 & 1.58 & 8.42 & $0.020 \S$ & 1.93 & 0.19 \\
\hline $\begin{array}{l}\text { Beta } \\
(12-30 \mathrm{~Hz})\end{array}$ & -1.29 & 0.26 & 0.62 & 0.59 & 0.53 & 0.71 & 7.42 & $0.026 \S$ & 1.83 & 0.2 \\
\hline $\begin{array}{l}\text { Gamma1 } \\
(30-58 \mathrm{~Hz})\end{array}$ & $2.62 \S$ & 4.10 & 0.08 & 1.47 & 0.26 & $2.96 \S$ & 1.80 & 0.22 & 1.04 & 0.37 \\
\hline $\begin{array}{l}\text { Gamma2 } \\
(62-100 \mathrm{~Hz})\end{array}$ & $6.3 \ddagger$ & 0.01 & 0.91 & 1.11 & 0.34 & $3.16 \S$ & 6.11 & $0.039 \S$ & 13.24 & $0.001 \ddagger$ \\
\hline
\end{tabular}

For each site (frontal cortex or hippocampus), (Walk-Imm) $t$ indicates the $t$-statistic for the power difference between walking and baseline immobility (Imm), using a combined group of lesioned and control rats $(n=10)$, and $F$ and probability $(P)$ of the null hypothesis for Group and Dose after two-factor repeatedmeasures ANOVA ( 2 groups $\times 3$ isoflurane doses) are listed. The two groups are septal cholinergic lesioned and control groups, each group consisting of five rats, and three doses $(0.5,0.625$, and $0.75 \%$ isoflurane).

* Theta band was 4-12 Hz for frontal electroencephalogram, and 7-8 Hz for hippocampal electroencephalogram. † $P$ values after repeated doses were adjusted by the Greenhouse-Geisser epsilon. $\ddagger P<0.005$. $§ P<0.05$. P values for group $\times$ dose interaction are not significant $(P>0.38)$, and not shown. $\mathrm{EEG}=$ electroencephalogram.

Hippocampal delta power was not significantly groupor isoflurane- dose-dependent (fig. 4A; table 1). In control rats, hippocampal theta $(7-8 \mathrm{~Hz}$; fig. $4 \mathrm{~A})$ and beta power (fig. 4B) decreased greatly with isoflurane dose in contrast to very small dose-dependent changes in lesioned rats (table 1). Hippocampal $\gamma 2$ power in all rats decreased significantly from baseline immobility to $0.5-0.75 \%$ isoflurane concentration, with lesioned rats showing a greater decrease in $\gamma 2$ power than control rats (fig. $4 \mathrm{C}$ and table 1 ). Hippocampal $\gamma 1$ power also decreased significantly from baseline immobility to $0.5 \%$ isoflurane concentration, but did not significantly change with higher isoflurane doses, or between lesioned and control groups (fig. 4C and table 1).

\section{Medial Septal Cholinergic Lesion Did Not Change Anesthetic Sensitivity to Ketamine}

The cumulative dose of ketamine that induced LORR was similar between lesioned and control rats. LORR occurred with ketamine dose of $112 \pm 21$ and $116 \pm 19 \mathrm{mg} / \mathrm{kg}, \mathrm{n}=5$ each, for lesioned rats and control rats, respectively $(P>0.8$, $t$ test). As estimated from the percent LORR versus ketamine dose graph (not shown), control rats gave an ED50 (LORR) of $103.1 \mathrm{mg} / \mathrm{kg}$ (95\% CI, 87.6-121.5 mg/kg), which was not significantly different from $105.1 \mathrm{mg} / \mathrm{kg}$ (95\% CI, $94-117.4 \mathrm{mg} / \mathrm{kg}$ ) in lesioned rats.

\section{Discussion}

We showed that rats with selective lesion of medial septal cholinergic neurons, as compared with sham-lesioned control rats, had a lower ED50 (LORR), shorter induction time, and longer emergence time associated with isoflurane anesthesia. The sensitivity to ketamine anesthesia was not different between lesioned rats and control rats. Hippocampal $\gamma 2(62-100 \mathrm{~Hz})$ power decreased with isoflurane concentration in a dose-dependent manner in both lesioned and control rats. However, the sensitivity of hippocampal $\gamma 2$ power to isoflurane was greater in lesioned than control rats, in correspondence with the higher LORR sensitivity to isoflurane in lesioned than control rats.

The current study underscores the importance of the septohippocampal system in general anesthesia. Laalou et al. ${ }^{13}$ reported that selective cholinergic lesion of the medial septum increased the anesthesia sensitivity to intraperitoneal propofol. The current study provides evidence that the sensitivity to isoflurane anesthesia was significantly increased by selective lesion of the cholinergic neurons in the medial septum, and ED50 (LORR) was shifted from $0.74 \%$ in shamlesioned rats to $0.62 \%$ in septal cholinergic lesioned rats. Because an inhaled volatile anesthetic could readily achieve equilibrium with concentrations in the alveoli and the brain, the current study provides a robust demonstration that isoflurane anesthesia sensitivity is different between septal cholinergic lesioned rats and control sham-lesioned rats.

In a previous study, we reported that the ED50 (LORR) was shifted from $1.02 \%$ in septal implanted control rats to $0.90 \%$ after electrolytic lesion of the medial septum. ${ }^{15}$ Although different equipment were used in the two studies, the low ED50 (LORR) in the current study suggests that sham lesion of the medial septum may have an effect in decreasing ED50 (LORR) as compared with septal implanted or unoperated rats. Previously, we reported that muscimol inactivation ${ }^{14}$ or electrolytic lesion ${ }^{15}$ of the medial septum prolonged the emergence from anesthesia induced by volatile or injectable anesthetics, which is consistent with the prolonged emergence from isoflurane reported here. 


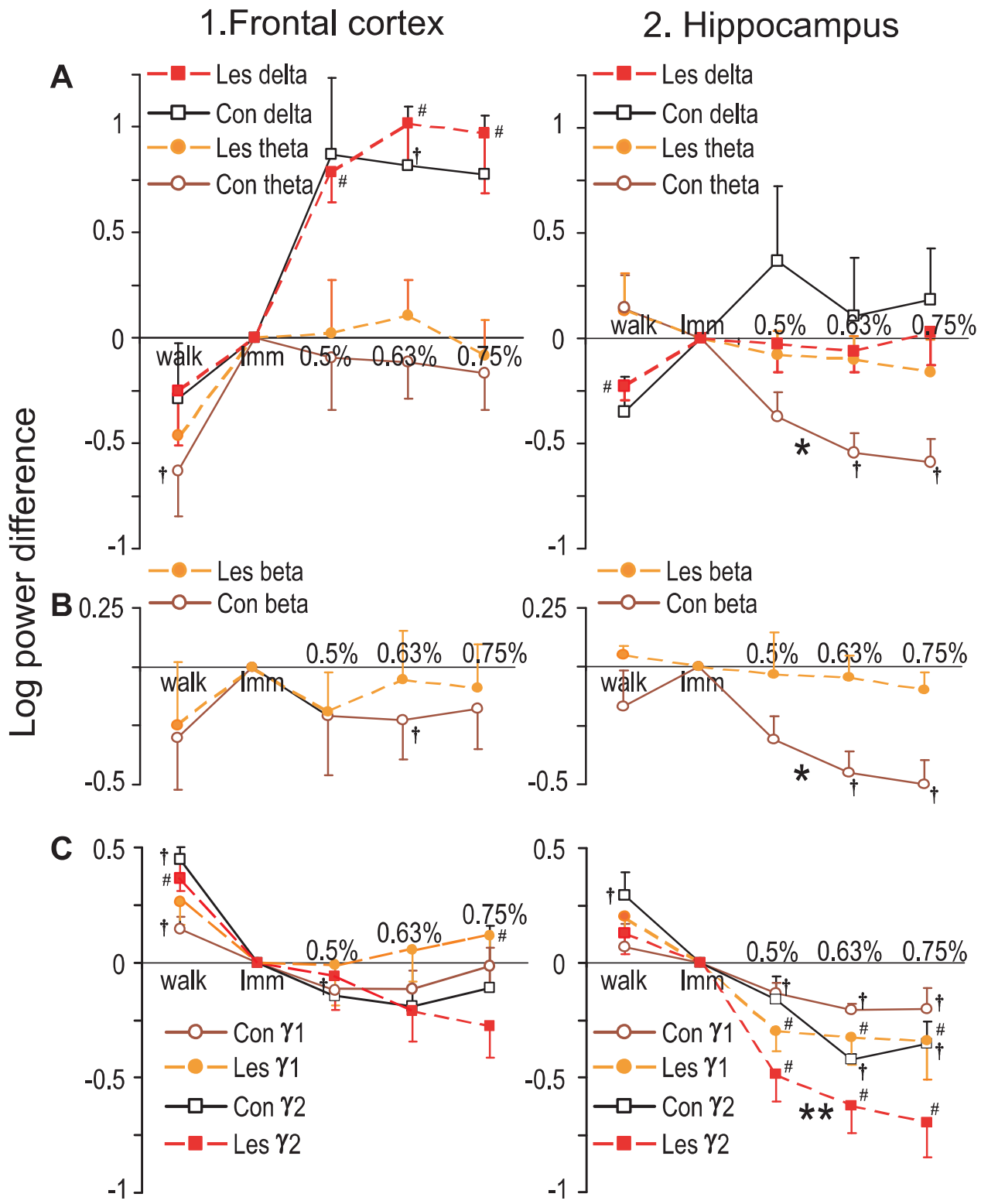

Fig. 4. Change in integrated power of electroencephalogram frequency bands (mean $\pm \mathrm{SEM}$ ) in the frontal cortex (left, column 1) and hippocampus (right, column 2) for different frequency bands (delta, theta, beta, low gamma $\gamma 1$, high gamma $\gamma 2$ ) in 192 IgG-saporin lesioned (Les) and sham-lesioned control (Con) groups of rats. Power change for each frequency band was the integrated logarithmic power of a condition minus the integrated logarithmic power during no-drug immobility baseline (Imm), and plotted horizontally from left to right: walk before isoflurane, Imm, and $15 \mathrm{~min}$ after $0.5,0.625$, and $0.75 \%$ isoflurane concentration. (A) Left, frontal delta power increased by $0.5 \%$ isoflurane in both lesioned and control rats, but not significantly isoflurane dosedependent. Frontal theta power did not change significantly with isoflurane doses. Right, hippocampal delta was not significantly different between groups, whereas hippocampal theta decreased with isoflurane dose only in control but not lesioned group. (B) Frontal beta power (left) was not significantly altered by isoflurane, whereas hippocampal beta (right) decreased with isoflurane dose only in control but not lesioned group. $(C)$ Left, frontal $\gamma 1(30-58 \mathrm{~Hz})$ and $\gamma 2(62-100 \mathrm{~Hz})$ were higher during baseline walk than Imm, but not significantly different between groups or isoflurane doses. Hippocampal $\gamma 1$ or $\gamma 2$ power (right) was generally higher during walk than Imm. $\gamma 2$, but not $\gamma 1$, was isoflurane-dose dependent and significantly different between groups. ${ }^{\star \star} P<0.01,{ }^{\star} P<0.05$, isoflurane response was significantly different between lesioned and control groups, two-factor repeatedmeasures ANOVA. $\dagger P<0.05$, control group, different from baseline Imm; $\# P<0.05$, lesioned group, different from baseline Imm.

However, quantitative comparisons of cholinergic lesioned rats with muscimol-inactivated or electrolytically lesioned rats could not be made because the procedures (dose and duration of isoflurane) used for induction and emergence tests were not standardized.
General anesthetics and slow-wave sleep have been suggested to share similarities in the mechanism of loss of consciousness. ${ }^{19,23,26,27}$ However, the cholinergic medial septal neurons do not regulate wakefulness and sleep, as seen from the lack of change of sleep and wake patterns after septal 
192 IgG-saporin lesion. ${ }^{28}$ The cholinergic septohippocampal neurons are, however, critically involved in activation of the hippocampus. Cholinergic neurons in the basal forebrain fired maximally during active waking as compared with quiet waking or slow-wave sleep, ${ }^{7}$ causing more release of acetylcholine in the hippocampus during active than quiet waking. ${ }^{4,29}$ The hippocampus is proposed to initiate movements, partly by activating dopaminergic release in the nucleus accumbens. ${ }^{30}$ We suggest that there is a septohippocampal activation that normally opposes isoflurane anesthesia and promotes emergence from anesthesia. The latter septohippocampal activation is suggested to be deficient after lesion of cholinergic neurons in the medial septum. However, behavioral activation was not different between lesioned and control rats when assessed by an enhanced hippocampal and frontal gamma power during walking as compared with immobility (fig. 4C), and lower hippocampal activation was suggested only by a smaller hippocampal theta rhythm during walking in lesioned as compared with control rats. ${ }^{20}$

This study reported that rats with lesion of septal cholinergic neurons showed shorter induction time, longer emergence time, and a leftward shift of the LORR-isoflurane dose-response curve as compared with control rats (fig. 2A). However, induction and emergence times may be differentially affected after a brain lesion. In mice with genetically ablated orexin neurons ${ }^{23}$ and in rats with histaminergic tuberomammillary nucleus lesion, ${ }^{25}$ emergence time was prolonged but induction time was similar when compared with the respective controls.

The current study confirmed and extended the relation of gamma activity with behavioral activation and general anesthesia. We showed that hippocampal and frontal gamma activity increased with behavioral activation ${ }^{1,29}$ and decreased with isoflurane anesthesia (fig. 4C). Previous studies showed that the high-frequency hippocampal gamma $(\gamma 2)$ was suppressed during deep anesthesia with different anesthetics, including isoflurane, halothane, propofol, and pentobarbital, ${ }^{14,24}$ and human temporal lobe gamma electroencephalogram was suppressed with sevoflurane anesthesia. ${ }^{31}$ The current study showed an isoflurane dose dependence for the hippocampal but not frontal high-frequency gamma, whereas Hudetz et al., ${ }^{32}$ using bilateral epidural recordings from the frontal cortex and a larger dose range of $0-1.2 \%$ isoflurane, showed that high-frequency $(70-140 \mathrm{~Hz})$ gamma decreased with isoflurane dose in both the frontal cortex and hippocampus. A lack of change of low-frequency gamma in frontal cortex and hippocampus with isoflurane was also reported by Hudetz et al. ${ }^{32}$ Other than gamma, large slow delta power in the frontal and hippocampal electroencephalogram accompanied hypnotic doses of isoflurane (figs. 3 and 4 ), but the delta power was not significantly dose dependent. However, hippocampal theta and beta power decreased with isoflurane dose in control but not lesioned rats (fig. 4, A and B), suggesting that an isoflurane-sensitive, low-frequency electroencephalogram component (theta and beta) was not present in lesioned rats.

Lesioned rats, as compared with control sham-lesioned rats, showed a stronger decrease in hippocampal $\gamma^{2}$ power at $0.5-0.75 \%$ isoflurane. However, baseline gamma (including $\gamma^{2}$ ) power was not significantly different between septal cholinergic lesioned and control rats, confirming previous studies. ${ }^{20,21}$ The sensitivity of hippocampal $\gamma^{2}$ power to isoflurane appears to indicate anesthesia (LORR) sensitivity. Lesioned rats showed a greater isoflurane-induced decrease in hippocampal $\gamma^{2}$ power than control rats, in correspondence with a lower isoflurane ED50 (LORR) in lesioned as compared with control rats.

Septal cholinergic lesion failed to affect LORR response to ketamine. This suggests that the anesthesia actions of ketamine and isoflurane were different, as has been suggested in other studies. ${ }^{25}$ In particular, selective lesion of $\gamma$-aminobutyric acidergic neurons in the medial septum suppressed the behavioral hyperactivity induced by ketamine, ${ }^{33}$ whereas selective lesion of cholinergic neurons did not alter behavioral hyperactivity or hippocampal gamma induced by another $N$-methylD-aspartate receptor antagonist phencyclidine. ${ }^{21}$ This suggests that ketamine actions on behavioral hyperactivity and LORR are not critically dependent on septal cholinergic neurons.

Our study has several limitations. First, the extent of cholinergic dysfunction in $192 \mathrm{IgG}$-saporin lesioned rats was not fully established. In rats with $192 \mathrm{IgG}$-saporin lesion, administration of anticholinesterase physostigmine could induce acetylcholine release, ${ }^{34}$ which may suggest function of the medial septal cholinergic neurons despite a near-complete lack of choline acetyltransferase-immunoreactive septal neurons. However, because the current study already found robust differences in isoflurane anesthesia response between 192 IgG-saporin lesioned rats as compared with control rats, it is possible that complete depletion of acetylcholine release in the hippocampus may have even more severe effects. Second, brain isoflurane concentrations, which may determine LORR, were not measured. Third, minute ventilation rate and respiratory rate during induction and emergence from isoflurane were not determined; respiratory rate may affect induction or emergence times, but probably not ED50 (steady-state) determination. Fourth, isoflurane was the only inhalational anesthetic used, and a general role of septal cholinergic neurons in mediating hypnosis by other inhalational anesthetics remains to be studied. Fifth, the determination of LORR after ketamine was difficult because at the point of LORR, as operationally defined earlier, a typical rat was able to turn its head and body in a supine position, without landing on its feet. In addition, cumulative intraperitoneal doses of ketamine were expected to show complex pharmacokinetics such that interpretation of results determined by cumulative intraperitoneal doses must be taken with caution.

Decline in cholinergic neurons in both the medial septum and nucleus basalis occurs during aging and more drastically in Alzheimer disease in humans. ${ }^{35}$ On the basis of the strong 
effects on anesthesia response caused by selective decrease of septal cholinergic neurons in the current study, it may be suggested that natural or pathological decrease in septal cholinergic neurons will contribute to the anesthesia sensitivity in the aged population ${ }^{36}$ and in Alzheimer patients.

In summary, the current results suggest that medial septal cholinergic neurons are involved in the modulation of isoflurane anesthesia. The results also suggest that hippocampal gamma activation is an indicator of the anesthesia effect of isoflurane, and the neural circuit for isoflurane anesthesia may differ from that for ketamine anesthesia.

\section{Acknowledgments}

The authors thank Stefanie Mok and Asfandyar Mufti, Department of Physiology and Pharmacology, The University of Western Ontario, London, Ontario, Canada, for their technical assistance.

This research was supported by the Canadian Institutes of Health Research (Grant MOP-15685) and Natural Science and Engineering Research Council (Grant 1037-2008), Ottawa, Ontario, Canada.

\section{Competing Interests}

The authors declare no competing interests.

\section{Correspondence}

Address correspondence to Dr. Leung: Department of Physiology and Pharmacology, The University of Western Ontario, London, Ontario N6A5C1, Canada. sleung@uwo.ca. Information on purchasing reprints may be found at www. anesthesiology.org or on the masthead page at the beginning of this issue. Anesthesiology's articles are made freely accessible to all readers, for personal use only, 6 months from the cover date of the issue.

\section{References}

1. Jones BE: Modulation of cortical activation and behavioral arousal by cholinergic and orexinergic systems. Ann N Y Acad Sci 2008; 1129:26-34

2. Phillis JW: Acetylcholine release from the central nervous system: A 50-year retrospective. Crit Rev Neurobiol 2005; $17: 161-217$

3. Marrosu F, Portas C, Mascia MS, Casu MA, Fà M, Giagheddu M, Imperato A, Gessa GL: Microdialysis measurement of cortical and hippocampal acetylcholine release during sleepwake cycle in freely moving cats. Brain Res 1995; 671:329-32

4. Dudar JD, Whishaw IQ, Szerb JC: Release of acetylcholine from the hippocampus of freely moving rats during sensory stimulation and running. Neuropharmacology 1979; 18 : 673-8

5. Saper CB: Organization of cerebral cortical afferent systems in the rat. II. Magnocellular basal nucleus. J Comp Neurol 1984; 222:313-42

6. Semba K: Multiple output pathways of the basal forebrain: Organization, chemical heterogeneity, and roles in vigilance. Behav Brain Res 2000; 115:117-41

7. Lee MG, Hassani OK, Alonso A, Jones BE: Cholinergic basal forebrain neurons burst with theta during waking and paradoxical sleep. J Neurosci 2005; 25:4365-9

8. Vanderwolf $\mathrm{CH}$ : Cerebral activity and behavior: Control by central cholinergic and serotonergic systems. Int Rev Neurobiol 1988; 30:225-340
9. Dong HL, Fukuda S, Murata E, Zhu Z, Higuchi T: Orexins increase cortical acetylcholine release and electroencephalographic activation through orexin-1 receptor in the rat basal forebrain during isoflurane anesthesia. ANESTHESIOLOGY 2006; 104:1023-32

10. Meuret P, Backman SB, Bonhomme V, Plourde G, Fiset P: Physostigmine reverses propofol-induced unconsciousness and attenuation of the auditory steady state response and bispectral index in human volunteers. ANESTHESIOLOGY 2000; 93:708-17

11. Hudetz AG, Wood JD, Kampine JP: Cholinergic reversal of isoflurane anesthesia in rats as measured by cross-approximate entropy of the electroencephalogram. Anesthesiology 2003; 99:1125-31

12. Leung LS, Petropoulos S, Shen B, Luo T, Herrick I, Rajakumar $\mathrm{N}, \mathrm{Ma} \mathrm{J}$ : Lesion of cholinergic basal forebrain neurons enhances the response to general anesthetics. Exp Neurol 2011; 228:259-69

13. Laalou FZ, de Vasconcelos AP, Oberling P, Jeltsch H, Cassel JC, Pain L: Involvement of the basal cholinergic forebrain in the mediation of general (propofol) anesthesia. Anesthesiology 2008; 108:888-96

14. Ma J, Shen B, Stewart LS, Herrick IA, Leung LS: The septohippocampal system participates in general anesthesia. J Neurosci 2002; 22 (RC200):1-6

15. Leung LS, Ma J, Shen B, Nachim I, Luo T: Medial septal lesion enhances general anesthesia response. Exp Neurol 2013; 247:419-28

16. Amaral DG, Kurz J: An analysis of the origins of the cholinergic and noncholinergic septal projections to the hippocampal formation of the rat. J Comp Neurol 1985; 240:37-59

17. Sotty F, Danik M, Manseau F, Laplante F, Quirion R, Williams $S$ : Distinct electrophysiological properties of glutamatergic, cholinergic and GABAergic rat septohippocampal neurons: Novel implications for hippocampal rhythmicity. J Physiol 2003; 551(Pt 3):927-43

18. Colom LV, Castaneda MT, Reyna T, Hernandez S, GarridoSanabria E: Characterization of medial septal glutamatergic neurons and their projection to the hippocampus. Synapse 2005; 58:151-64

19. Franks NP: General anaesthesia: From molecular targets to neuronal pathways of sleep and arousal. Nat Rev Neurosci 2008; 9:370-86

20. Lee MG., Chrobak JJ, Sik A, Wiley RG, Buzsáki G: Hippocampal theta following selective lesion of the septal cholinergic system. Neuroscience 1994; 62:1033-47

21. Ma J, Shen B, Rajakumar N, Leung LS: The medial septum mediates impairment of prepulse inhibition of acoustic startle induced by a hippocampal seizure or phencyclidine. Behav Brain Res 2004; 155:153-66

22. Paxinos G, Watson C: The Rat Brain in Stereotaxic Coordinates, 4th edition. New York, Academic Press, 1998

23. Kelz MB, Sun Y, Chen J, Cheng Meng Q, Moore JT, Veasey SC, Dixon S, Thornton M, Funato H, Yanagisawa M: An essential role for orexins in emergence from general anesthesia. Proc Natl Acad Sci U S A 2008; 105:1309-14

24. Leung LS, Lopes da Silva FH, Wadman WJ: Spectral characteristics of the hippocampal EEG in the freely moving rat. Electroencephalogr Clin Neurophysiol 1982; 54:203-19

25. Luo T, Leung LS: Basal forebrain histaminergic transmission modulates electroencephalographic activity and emergence from isoflurane anesthesia. ANESTHESIOLOGY 2009; 111: 725-33

26. Lydic R, Baghdoyan HA: Sleep, anesthesiology, and the neurobiology of arousal state control. AnESTHESIOLOGY 2005; 103:1268-95

27. Lu J, Nelson LE, Franks N, Maze M, Chamberlin NL, Saper CB: Role of endogenous sleep-wake and analgesic systems in anesthesia. J Comp Neurol 2008; 508:648-62 
28. Blanco-Centurion C, Gerashchenko D, Shiromani PJ: Effects of saporin-induced lesions of three arousal populations on daily levels of sleep and wake. J Neurosci 2007; 27: 14041-8

29. Leung LS: Generation of theta and gamma rhythms in the hippocampus. Neurosci Biobehav Rev 1998; 22:275-90

30. Mogenson GJ, Brudzynski SM, Wu M, Yang CR, Yim CY: From motivation to action: A review of dopaminergic regulation of limbic-nucleus accumbens-ventral pallidum-pedunculopontine nucleus circuits involved in limbic-motor integration, Limbic Motor Circuits and Neuropsychiatry. Edited by Kalivas PW, Barnes CD. Boca Raton, CRC Press, 1993, pp 193-225

31. Uchida S, Nakayama H, Maehara T, Hirai N, Arakaki H, Nakamura M, Nakabayashi T, Shimizu H: Suppression of gamma activity in the human medial temporal lobe by sevoflurane anesthesia. Neuroreport 2000; 11:39-42
32. Hudetz AG, Vizuete JA, Pillay S: Differential effects of isoflurane on high-frequency and low-frequency $\gamma$ oscillations in the cerebral cortex and hippocampus in freely moving rats. ANESTHESIOLOGY 2011; 114:588-95

33. Ma J, Tai SK, Leung LS: Septohippocampal GABAergic neurons mediate the altered behaviors induced by n-methyl-D-aspartate receptor antagonists. Hippocampus 2012; 22:2208-18

34. Chang Q, Gold PE: Impaired and spared cholinergic functions in the hippocampus after lesions of the medial septum/vertical limb of the diagonal band with 192 IgG-saporin. Hippocampus 2004; 14:170-9

35. Guela C, Mesulam MM: Cholinergic system and related neuropathological predilection patterns in Alzheimer disease, Alzheimer Disease. Edited by Terry RD, Katzman R, Bick KL. New York, Raven Press, 1994, pp 263-92

36. Vuyk J: Pharmacodynamics in the elderly. Best Pract Res Clin Anaesthesiol 2003; 17:207-18 